\title{
NEOLOGISMI TECNICI ED AFFINI NELLA PROSA ROVIGNESE ATTUALE
}

1. La lingua delle opere in rovignese, apparse sulle pagine dell'antologia Istria Nobilissima, offre materiali ricchi e svariati per diversi tipi di studi linguistici, fra i quali quello del livello lessicale è uno dei più importanti. Si sa, infatti, che proprio nel lessico, meglio che nei livelli fonologico e morfosintattico, si riflettono le vicende di un idioma e della comunità che lo parla. Dopo avere studiato il lessico della prosa rovignese nella citata antologia da vari punti di vista (Tekavčić 1983a, 1983b, 1984a, 1984b, Elemento tedesco, Tekavčić Riflessi), abbiamo deciso di aggiungere qualche pagina su un aspetto forse minore, ma certamente non privo di importanza, del patrimonio lessicale del rovignese: $i$ neologismi, prevalentemente nel settore tecnico ma anche in certi altri settori affini. Poiché la linguistica attuale non si limita, come quella di un tempo, ai settori ritenuti «genuini», «puri», «conservatori» ecc., ma studia l'idioma tutto quanto, tale quale funziona nella sua comunità in tutte le occasioni e a tutti i fini, è logico che anche i neologismi vi trovino posto; anzi, sono proprio essi che provano la capacità creativa, innovativa, di un idioma e con ciò anche la sua vitalità. Si confrontino recentemente i contributi di E. Diekmann sulla terminologia dell'automobilismo e dei settori lessicali connessi (Diekmann (1983, 1984).

2. Il nostro argomento impone di restringere il corpus alla produzione in prosa, che si trova nei volumi III, VI, VII, X, XI, XII, XIV, XV e XVI dell'antologia Istria Nobilissima ${ }^{1}$, mentre solo in via del tutto eccezionale vi abbiamo aggiunto brani verseggiati (praticamente, soltanto la poesia La farata di G. Curto, vol. IV, pp. 244-245). Abbiamo ottenuto cosí un corpus di 280 pagine cca, in cui sono stati riscontrati una ventina di neologismi (prevalentemente tecnici, alcuni appartenenti allo sport e agli armamenti). I testi sono opera di tre autori (Giusto Curto, Giovanni Pellizzer, Giovanni Santin); la maggioranza ne sono dialogati (essendo destinati alla scena: bozzetti, brevi commedie ecc.), solo alcuni sono in prosa, di carattere narrativo (ricordi, novelline, storielle ecc.) e l'argomento è vario; insomma, possono essere considerati rappresentativi del dialetto istroromanzo rovignese (nella misura, ovviamente, in cui un testo letterario può mai essere rappresentativo del linguaggio spon-

1 Abbiamo spogliato gli stessi testi come negli studi precedenti, e cioè: vol. III: G. Pellizzer, El spacio da sa Bunita (pp. 199-215); vol. VI: G. Curto, Zi muorta sa Batalita (pp. 63-78); vol. VII: G. Santin, Leggende e novelle antiche (pp. 151-201), G. e A. Pellizzer, Sango nusento (pp. 209-225, solo parzialmente in rovignese); vol. X: G. Curto, El spuzaleîsio in furno da sà Mareîa furniera (pp. 129-158); vol. XI: G. Curto, Meînguele [sic; = Meingule] ingrumade (pp. 143-152); vol. XII: G. Pellizzer, Chế fa carta in veîta, moro in sufeîta (pp. 239-261), G. Curto, El bateîzo (pp. 263-318); vol. XIV: G. Pellizzer, Stuorie da pascaduri da cuntaghe ai nevi (pp. 111-138); vol. XV: G. Curto, Quil malagnazo viazo da Tristi (pp. 153-191); vol. XVI: G. Curto, Meîle nuò pioûn meile (pp. 131-158). 
taneo). Il numero relativemente scarso dei neologismi è in rapporto con il contenuto ed il carattere generale dei testi: essi tendono ad illustrare l'ambiente familiare di un tempo, le tradizioni, gli usi di una volta, la vita nella Rovigno di molti anni fa ecc., sicché poco si prestano all'uso dei neologismi.

Il nostro studio dei neologismi in rovignese si articola nei seguenti tre punti: esempi (tradotti e ove occorre commentati), esame semantico, esame formale (adattamenti fonetici).

\section{Esempi:2}

\section{1 auto 'auto':}

(1) tu mare Marioûsa la zi zeîda a la curiera a vidi sa ti rê̂vi [. . .]. - Invise cume ch'i vadi siemo vignoûdi cu l'auto. (XV, 167) 'tua madre Mariuccia è andata alla corriera a vedere se arrivi [. . . ]. - Invece, come vedete, siamo venuti con la macchina.'

(2) pruopio in quil mumento a gira rivà l'urdane da rinvià i lavuri, e cusế [...] $i$ vemo ciapà l'auto e i sa la vemo mucada. $(\mathrm{XV}, 169)$ 'proprio in quel momento era arrivato l'ordine di rinviare i lavori, e così [. . . ] abbiamo preso la macchina e ce la siamo svignata.'

3.2 bapur 'pentola a pressione in ghisa usata in passato'. ${ }^{3}$

(3) Dame qua el bapur ca nu zì tenpo da mignulasala. (XII, 218) 'Dami qua la pentola [con l'acqua bollente], ché non c'è tempo per indugiare'.

(4) (un vecchio pescatore, che crede nelle streghe, racconta di esse) tri da lure li $\int_{i}$ feîde in sulier [...] quile altre [...] il uò ruto dô̂ti i piati e, cume sa nu bastiso, in oûltima li uò pisà in bapur. (XIV, 133) 'tre di esse andarono in soffitta [. . . ] quelle altre ruppero tutti i piatti e, come se ciò non bastasse, in fine pisciarono nella pentola a pressione."

\section{3 binario 'binario':}

(5) Zi sta ligreîa, ma pioûn calvario/su 'l tuovo oûnico binario. (IV, 245)'C'è stata allegria, ma più calvario sul tuo unico binario' (traduzione dell'autore).

(6) Fếla par el tuovo binario, Latansia, ca meîo la scola i la iè fata da peîcia [. . . ] ca nù ma curo maiestre [. . . ] (XII, 273) 'Fila per il tuo binario, Lattanzia, ché io la scuola l'ho fatta da piccola [. . . ] sicché non mi occorrono maestre [. . . ]"

(7) Nu sta farmate mità binario, spoûda el'oûso . . . Ch'i zilo? (XV, 164) 'Non fermarti a metà binario, sputa l'osso . . . Che cosa c'è?‘

2 Gli esempi vengono citati nella grafia originale, e i nostri interventi (tra parentesi quadre) sono limitati alle correzioni e spiegazioni indispensabili; inoltre, abbiamo corretto errori tipografici evidenti. Gli accenti usati dagli autori (quasi sempre in modo arbitrario ed impressionistico) vengono riprodotti nelle citazioni, non nelle analisi ulteriori. L'accento tonico viene indicato solo là dove ci. potrebbero essere dubbi sulla sua posizione, e unicamente con l'accento grafico acuto. - Gli esempi vengono seguiti dall'indicazione del volume di Istria Nobilissima (in cifre romane) e della pagina (in cifre arabe). Spegazione dell'autore G. Pellizzer, XIV 133, nota 13. 


\section{4 bulitur 'bollitore':}

(7a) In quila ticia [. . .] la cuziniva el magnà da zura d’oun bulitùr a gaz [. . .] (VII, 169) 'In quel tegame [. . .] essa cucinava il mangiare su un bollitore a gas $[. .]^{6}$

\section{5 cine 'cinema':}

(8) (ricordi della vita d'un tempo) E cine? I vularàvi vidi ca cagnara, duopo el preimo ato [. . .] (VII, 178) ' $\mathrm{E}$ il cinema? Vorrei vedere che cagnara, dopo il primo tempo [... $]^{6}$

3.6 curiera 'corriera, pullman di linea':

(9) ti fariè tardi a la curiera, la pol rivà in anteîcipo a logo. - Da quando in qua li curiere rê̂va in antếcipo [. . .] $(\mathrm{XV}, 165)$ 'farai tardi alla corriera, essa può anche arrivare in anticipo. - Da quando in qua le corriere arrivano in anticipo $[. . .]^{\text {s }}$

3.7 farata'(strada) ferrata, ferrovia':

(10) Farata . . . quando ch'i ta uò inagurada / ti giri maistuza e doûta inbandara$d a[\ldots](I V, 244)$ 'Strada ferrata . . . quando t'hanno inaugurata / eri maestosa, tutta imbandierata [. . . ]' (traduzione dell'autore)

(11) ti sufi cume la farata ca va soûn par Marbuoi [. . .] (XII, 267) 'sbuffi come la ferrovia che sale per Marbuoi [. . .]“

(12) (parole rivolte ad una vecchia che critica il mondo moderno e specialmente la gioventù) Sa Cucalita, el mondo cameîna, cameîna la farata, el nutunobile [. . . ] e sa vantagia anche la zuvintoû [. . .] (XII, 303) 'Sora Cocaletta, il mondo cammina (= progredisce), cammina la ferrovia, l'automobile [. . .] e ne approfitta anche la gioventù [. . . ]

(13) oûna [delle due figlie] la zi a Zagabria spuzada c'oûn machineîsta da li farate [. . .] $(\mathrm{XV}, 187)$ 'una è a Zagabria, sposata con un macchinista delle ferrovie $[. .]^{6}$

\section{8 futubal 'calcio':}

(14) (anche qui: descrizione della vita passata, come nell'es. 8): Poupi e pinicule tajade cui arteisti del cine o zugaduri del futubal [. . .] (VII, 178) 'Puppi e pellicole $(=$ film) tagliate con artisti del cinema o giocatori di calcio [. . . .'

3.9 lavatreîce 'lavatrice' (elettrodomestico):

(15) Ca biancareîa candada, ch'i pulvare ti duopri? . . . - Par dête la verità i nu lu siè, ma lava ma feîa Piareîna cu la lavatreîce [. . .] (XVI, 144) 'Che biancheria candida, che polvere $[=$ detersivo] adoperi? . ...- Per dirti la verità, non lo so, mi lava mia figlia Pierina con la lavatrice $[\ldots]^{6}$ 
3.10 lietrico 'casco dei parrucchieri' (es. 16); 'energia elettrica' (es. 17):

(16) Là paron Giuvani [il parrucchiere] [. . .] el fiva el su'master culi fimane incapalade dazuta del liètrico [...] (VII, 162) 'Là padron Giovanni [...] faceva il suo mestiere con le donne incappellate sotto il casco [.... $]^{\circ}$

(17) Doûta quila ruoba mudierna ca va vanti a lietrico iè pagoûra ca scupio... (XVI, 144) 'Tutta quella roba moderna che funziona ad elettricità ho paura che scoppi ...

3.11 masinegaver, masinigaver 'mitragliatrice' $(<$ ted. Maschinengewehr):

(18) (detto ironicamente di una pettegola): Ara ca la zi cume oûna masinigaver: $n u$ la faleîso un culpo [. . .] $(\mathrm{XV}, 161)$ 'Guarda che [essa] è come un mitra: non fallisce un colpo'

(19) par gangade e fraîte el gira oûn masinegaver [. . . (XVI, 148) 'per le feste e le baldorie egli era un mitra [. . . $]^{6}$

3.12 machinario 'macchina, motore' (es. 20); 'apparecchio (es. 21):

(20) Ouu [. . .] farmì el machinario, ch'i dievo zmuntà. (XI, 150) 'Ehi [. . .] fermate la macchina, ché devo sbarcare.'

(21) A f ilo machinari sigoûri, fasile da duparali. (XVI, 144) 'Sono apparecchi sicuri, facile da adoperarli [ = 'facili da adoperare'o 'è facile adoperarli']

3.13 machineîsta 'macchinista':

v. 1'es (13).

3.14 mascara 'maschera' (per la pesca subacquea):

(22) A gira rivà là oun tureista [. . .] cum mascara e dardi par pascà zuta fondo. (VII, 190) 'Era venuto là un turista [. . . ] con maschera e dardi per pescare sott'acqua'.

3.15 muturein 'motoscafo':

(23) i zièmi [. . .] al mulito del laruojo, par spatà el muturein chel 'nda purtisso sul scujo da Santandrìa. (VII, 189) 'andavamo [. . .] al moletto dell'orologio ad. aspettare il motoscafo che ci portasse sullo scoglio di Sant'Andrea.'

3.16 natunobile 'automobile':

(24) quando el sabo sento li sone dei natunobili cui spugi [. . . ] a ma sa spalanca el cuor da giuoia . . . (XV, 161) 'quando il sabato sento i clacson delle macchine con gli sposi [...] mi si spalanca il cuore di gioia [...].‘

3.17 nutunobile 'automobile': v. l'es (12). 
3.18 pineicula 'pellicola, film':

(25) (nel cinema di un tempo) oun ciapo da muriedi [. . .] i sa cassutiva [. . . ] e . . . pache vire meinga par posta, cume intula pineicula! (VII, 178) 'una folla di ragazzi [...] si scazzottavano [. . .] e . . botte vere, mica finte come nei film!

(26) Sa in quil tenpo fuoso sta la tilivifion, i saravo vignô̂di a fâ la pinê̂cula. $(X I V, 114)$ 'Se in quei tempi ci fosse stata la televisione, sarebbero venuti a girare il film.'

3.19 pinicula 'pellicola, film':

v. l'es. (14).

3.20 saparneîn' 'shrapnel':

(27) (un vecchio pescatore si arrabbia contro un suo giovane collega) Quila granata ca nun ta vuò ciantrà in Galeîsia, quil saparneîn ca nun ta vuò zgnacà veîa el cavo [. . . ] ! (XI, 146) 'Quella granata che non ti ha centrato in Galizia, quello shrapnel che non ti ha portato via la testa [. . .]!‘

3.21 sipileîn 'zeppelin':

(28) (un po' dopo la scena precedente, il giovane marinaio, cacciato in terra dal vecchio, inveisce a sua volta contro Rovigno): Quil gianarà d'oûn can da Sipileîn giarmanico, ca nun uò bunbardà Ruveîgno [. . .] ! (XI, 147) 'Quel figlio d'un cane dello zeppelin germanico che non ha bombardato Rovigno [... $] !^{\bullet}$

3.22 taikefano, taliefono 'telefono':

(29) Basta [. . .] adieso el mieîo sacratario bataruò el taliefano a Ruveîgno [. . .]. Matate [= Metate] coûcio santà là, e sta bon feîn ca veîn la batoûda da taliefano da Ruveîgno. (XI, 152) 'Basta [. . .] adesso il mio segretario darà un colpo di telefono a Rovigno [. . . ]. Mettiti a sedere là e sta fermo finché viene il colpo di telefono da Rovigno‘.

(30) La murieda uò razon, quanto sa stiva daghe oûn culpo da taliefono [. . .] $(\mathrm{XV}, 167)$ 'La ragazza ha ragione, quanto ci si stava per darle un colpo di telefono [. . . $]^{6}$

(31) Sti [sic!; = Sti] poûr sierti ch'i nun bato taliefono puorta per puorta . . . (XVI, 147) 'State sicuri che io non faccio telefonate porta per porta . . .' (parole di una vecchia pettegola che, invece, delle «telefonate» ne fa, e come, sulle «porte» di tutto il vicinato ....)

\subsection{3 tilifon 'telefono':}

(32) ara ca quile patreîsie el suovo tilifon el rê̂va feîn in seîma al campaneîl. (XV, 184) 'Guarda che quelle patrizie il loro telefono arriva in cima al campanile.' 


\subsection{4 tilifunada 'telefonata':}

(33) sa duviva daghe oûna tilifunada [. . .] par nu fala stà cun travaîo. (XV, 167) 'le si doveva fare una telefonata [. . .] per non farla stare in pena.'

\subsection{5 tilivi Sion 'televisione':} v. l'es. (26).

\subsection{6 utumobile 'automobile‘:}

(34) gila [. . . ] la àbata in veîla [. . .] i vuò l'utumobile [. . .] (XVI, 144) 'essa [. . . ] abita in villa [. . .] hanno l'automobile [. . . $]^{6}$

(35) cume i uò pusiố fa quila biela vê̂la cun l'utumobile [. . .] Cume? (XVI, 145) 'come si sono potuti fare quella bella villa con l'automobile [. . . ] Come?'

\subsection{7 utunobile 'automobile':}

(36) loû uò l'utunobile [. . .] siemo capeîdi? (XV, 164) 'Egli ha la macchina [. . .] ci siamo capiti?'

(37) Lurenso ti son zeî ciula cu l'utunobile? (XV, 167) 'Lorenzo, sei andato a prenderla con la macchina?"

(38) Ah, ah, la foûga d'Agito cu l'utunobile [. . .] puovara muradola. (XV, 173) 'Ah, ah, la fuga d'Egitto in macchina [. . . ] povera ragazzina.'

\subsection{8 vagon 'vagone":}

(39) El cundutier su 'l vagon, inpirà cume oûna suvita, / ogni tanto el diva oûna sunada da trunbita. (IV, 244) 'Il conducente sul vagone, impettito come una civetta / ogni tanto suonava la trombetta.' (traduzione dell'autore)

\section{Esame semantico 4}

4.1 Come si è detto nell'introduzione, tutte le parole qui esaminate sono contraddistinte dal sema [moderno] (equivalente, nel nostro caso, su per giù agli ultimi cento anni o poco più), sicché esso non verrà più ripetuto nelle analisi e suddivisioni che seguono. In tutto il corpus dei nostri neologismi una prima distinzione separerà machineista, afetto dal tratto [ + umano], da tutto il resto, per il quale è allora valido [-umano]; in questo gruppo, caratterizzato dal sema [-umano], possiamo introdurre una seconda distinzione, basata sull'alternativa azione $+/-$ : tilifunada risponde con + , tutte le altre parole con - ('meno'). ${ }^{5}$ Le altre distinzioni che proponiamo sono queste: l'alternativa tecnica/sport (la risposta destra isolerà le voci futubal e mascara, per cui si può introdurre un'alternativa speciale attività/requisito; la

4 Adottiamo, con determinate modifiche, il procedimento di M. Alinei (1974).

5 Il derivato tilifunada ha un sinonimo in batoûda da taliefano, nell'esempio (29). 
risposta sinistra va suddivisa ulteriormente); la seguente alternativa potrà essere energia/applicazione, per separare lietricon (esempio 17) 'energia elettrica, corrente elettrica' da lietrico1 (esempio 16) ed altri vocaboli; l'applicazione della [tecnica] si suddivide a sua volta in base all'alternativa destinazione pacifica/bellica, che consente di opporre masinigaver (-egaver) e saparneîn (risposta destra) agli altri termini (risposta sinistra). A questo punto abbiamo enucleato su per giù il gruppo di 'macchine, apparecchi, utensili ecc. ' in senso più stretto, ma diverse alternative sono ancora necessarie: un'apposita alternativa, formulabile come specificato +1 - dividerà il termine machinario (risposta destra) dagli altri, le cui funzioni sono maggiormente specificate: l'alternativa macchine (ecc.) per locomozione/per altri scopi isolerà tutte le denominazioni dei mezzi di trasporto (risposta sinistra) dalle altre (risposta destra); i termini per i mezzi di trasporto si dividono in quelli su superficie terrestre (divisione ulteriore: a terra/su acqua, di cui la risposta destra vale per $m u$ turein, mentre la risposta sinistra richiede un'ulteriore distinzione, a seconda che si tratti di mezzi di trasporto a marcia guidata (binario, farata, vagone, divisibili in base ad alternative in cui non ci addentreremo più) o a marcia libera (auto e varianti)) e quelli in aria, rappresentati nel nostro corpus soltanto da sipileîn. Infine, le denominazioni delle macchine destinate ad altri scopi ammettono un'ultima alternativa: utensili, (elettro)domestici ecc./mezzi di comunicazione e/o di massa; la risposta sinistra raggruppa le voci bapur, bulitur, lavatreîce e lietrico1, quella destra racchiude invece cine, pineicula (-icula), taliefano (e varianti)e tilivision (divisibili in mezzi di comunicazione (taliefano) e mass media (cine, pineicula, tilivision).

4.2 Per rendere più chiara l'analisi semantica, la formuliamo nel seguente «albero rovesciato»: 
1. UMANO/NON UMANO

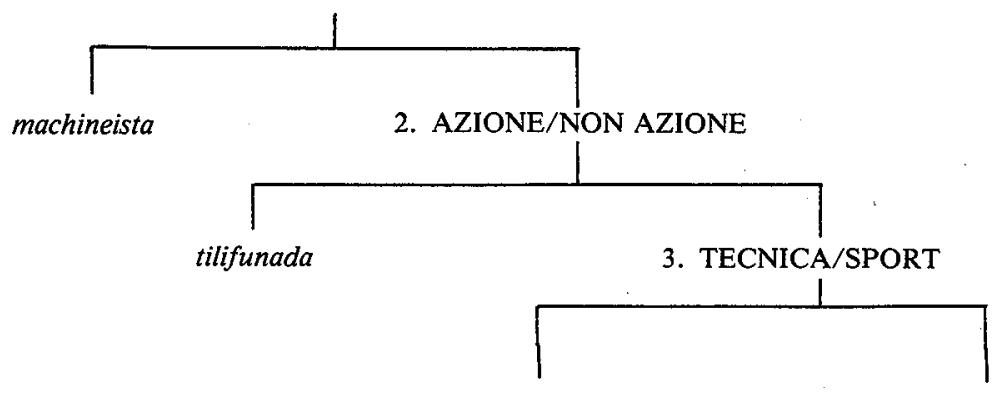

5. ENERGIA/APPLICAZIONE

lietrico2
4. ATTIVITÀ/REQUISITI

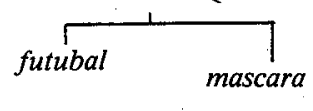

6. DESTINAZIONE

PACIF./BELL.

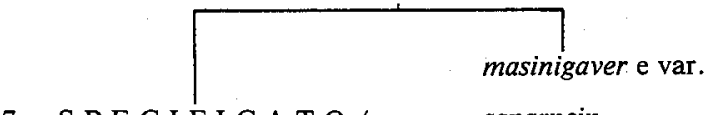

7. S P E C I F I C A T O/ saparnein NON SPECIFICATO

machinario

8. PER LOCOMOZIONE/

PER ALTRI SCOPI

10. IN SUPERFICIE TERRESTRE/

IN ARIA

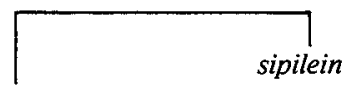

12. IN TERRA/SU ACQUA

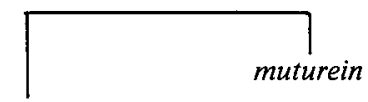

13. MARCIA

GUIDATA/LIBERA

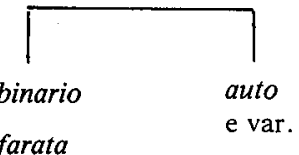

vagon
9. UTENSILI, (ELETTRO)DOMESTICI/ MEZZI DI COMUNICAZ., MASS MEDIA

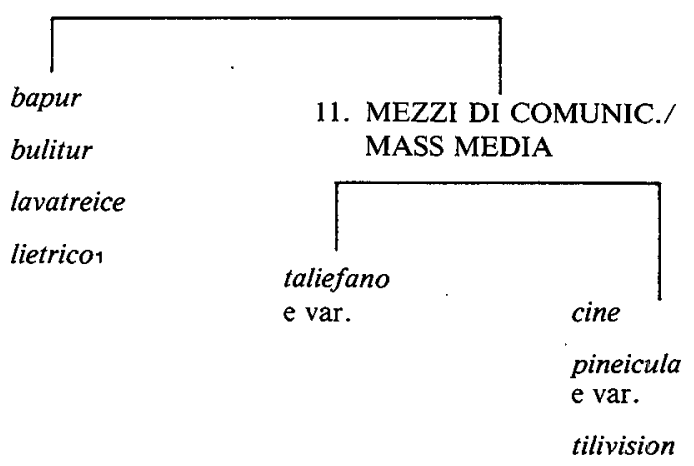


4.3 Da questo grafico si possono desumere le tormule della composizione semantica delle singole parole. Le diamo qui in forma alquanto semplificata:

machineista: [umano] (specificabile poi come [nome d'agente]),

tilifunada: [non umano] [azione]

futubal: [non umano] [non azione] [sport] [attività]

mascara: , [non umano] [non azione] [sport] [requisito]

lietrico2: [non umano] [non azione] [tecnica] [energia]

masinigaver: [non umano] [non azione] [tecnica] [applicazione] [destin. bellica]

e var.

saparnein: come il precedente ${ }^{6}$

machinario: [non umano] [non azione] [tecnica] [applicazione] [destin. pacifica] [non specificato]

bapur: [non umano] [non azione] [tecnica] [applicazione] [destinazione pacifica] [specificato] [non per locomozione] [utensili, (elettro)domestici]

bulitur: come il precedente

lavatreice: $\quad$ come i due precedenti

lietrico1: come $\mathrm{i}$ tre precedent $\mathrm{i}^{7}$

taliefano: [non umano] [non azione] [tecnica] [applicazione] [destin. pacifica] [non per locomozione] [mezzo di comunicazione]

e var.

cine: [non umano] [non azione] [tecnica] [applicazione] [destin. pacifica] [specificato] [non per locomozione] [mezzo di massa — mass medium]

pineicula: $\quad$ come il precedente

e var.

tilivision: come $\mathrm{i}$ due precedenti ${ }^{8}$

sipilein: [non umano] [non azione] [tecnica] [applicazione] [destin. pacifica] [specificato] [per locomozione] [in aria]

muturein: [non umano] [non azione] [tecnica] [applicazione] [destin. pacifica] [specificato] [per locomozione] [a livello della superficie terrestre] [su acqua]

auto e var.: [non umano] [non azione] [tecnica] [applicazione] [destin. pacifica] [specificato] [per locomozione] [a livello della superficie terrestre] [in terra] [marcia libera]

farata: [non umano] [non azione] [tecnica] [applicazione] [destinaz. pacifica] [specificato] [per locomozione] [a livello della superficie terrestre] [in terra] [marcia guidata]

6 Per distinguere i due termini bellici si può introdurre un'apposita alternativa pezzo d'artiglieria / proiettile, di cui però prescindiamo per non sovraccaricare l'analisi.

7 Anche qui si possono introdurre alternative inferiori: a corrente elettrica $\%$ a gas (o altro fuoco), per separare lavatreice e lietrico dagli altri due (per ciascuno dei due gruppi ci saranno alternative ulteriori ecc.).

8 Con suddivisioni anche qui. Aggiungiamo per completare che il rapporto fra pineicula e cine (o pineicula e tilivision) è analogo a quello tra binario e farata ('parte': 'tutto', sineddoche). 
binario: $\quad$ come il precedente (v. la nota 8 )

vagon: come i due precedenti.

4.4 Due parole ancora sulla vecchia ma a nostro avviso tuttora utile distinzione tra senso proprio e senso figurato (distinzione che ci pare utile soprattuto nell'analisi semantica di opere letterarie). A questo proposito si constata subito che tutte le nostre parole compaiono nei loro rispettivi sensi propri, e che soltanto due di esse, $b i$ nario e masinegaver (e precisamente in questa forma, non masinigaver), hanno a $\mathrm{n}$ $\mathrm{c} h$ e significati figurativi: binario negli esempi (6) e (7), masinegaver nell'esempio (19). Questa netta prevalenza dei sensi propri su quelli figurativi concorda con il semantismo dei termini tecnici in genere.

\section{Esame formale (adattamenti fonetici)}

5.1 Quello che in certi studi precedenti abbiamo constatato a proposito del trattamento degli elementi alloglotti in rovignese (e istroromanzo in genere) (cfr. Tekavčić 1984a, 1984b, Elemento tedesco) vale anche per i neologismi di significato tecnico e affine: anch'essi vengono «rovignizzati» mediante introduzione, spesso ipercoretta (ai fini dell'ipercaratterizzazione), delle caratteristiche fonetiche rovignesi. Gli adattamenti più importanti riposano tutti sulle evoluzioni fonetiche ben note nella grammatica storica istroromanza, in particolare rovignese. Si tratta dei seguenti processi.

5.2. Dittongamento dei fonemi romanzi /ę $g /$ in /ye wo/, dittonghi che ci conservano in sillaba chiusa, mentre tendono a monottongarsi in $/ \mathrm{i} u /$ in sillaba aperta (accrescendo così la frequenza dei fonemi vocalici chiusi, v. av.): PERDIT $>$ pierdo, FESTA > fiesta, FORTE > fuorto, FOSSA > fuosa , LEVAT > liva, DE$\mathrm{CE}(\mathrm{M})>$ gize, NOVU $>$ nuvo, MODU $>$ mudo ecc. I due dittonghi ascendenti vengono in seguito estesi anche ai contesti storicamente non giustificati: mieno 'meno', muona 'cretino' ecc. Il primo dei dittonghi appare nei nostri neologismi: lietrico, taliefano,-fono.

5.3 Ugualmente caratteristici per il rovignese sono i due dittonghi discendenti, /ey, ow/, provenienti dai fonemi romanzi /i u/ $(<$ class. $/ \overline{\mathrm{i}} \overline{\mathrm{u}} /)$, originariamente in sillaba libera: FILAT $>$ feila, -IRE $>-e i$, UNA $>$ ouna, MURU $>$ mouro ecc. Anche questi dittonghi vengono in seguito estesi ad altri contesti, ad esempio ai venetismi Dio e duto (acc. a tuto), da dove i rov. Deio, douto, nonché ai neologismi (ad es. i molti nomi moderni in -eista $<-i s t a$ ) e, nei testi contemporanei, anche ai fonemi /i u/provenienti dai romanzi /ẹ ọ/ (< class. /i $\overline{\mathrm{e}}$; u $\mathrm{o} /)$ per generalizzazione degli esiti originariamente metafonici (così ME(N)SE diventa regolarmente miz, per «iperdittongare» in meiz, e FLORE, attraverso il reg. fiur diventa fiour). Il primo dei due dittonghi è bene rappresentato nel nostro corpus: lavatreice, machineista, muturein, pineicula, saparnein, sipilein. ${ }^{9}$

9 Il fatto che di ambedue le coppie di dittonghi ricorra nel nostro corpus soltanto il membro contenente vocale e semivocale anteriori non può essere dovuto che ad un puro caso. 
5.4 Nell'evoluzione delle vocali protoniche si constata la coesistenza di due tendenze contrastanti: 1 ) la chiusura delle vocali romanze /e o/ in /i $u$ / (fenomeno ben noto dalla fonetica storica dell'italiano letterario e di numerosi dialetti italiani): SENTIRE $>$ sintei, MEDIETATE $>$ mitá, it. vestito $>$ visteito, ven. Betina $>B i$ teina; POTERE $>$ pudí, VOLERE $>$ vulí, CORONA $>$ kurona, it. dottore $>d u$ tur, ven. Tonin, $-a>$ Tunein, $-a$ ecc.; 2) l'apertura delle stesse due vocali e la loro convergenza in $/ a /$, frequente soprattutto per la /e/, meno per la /o/: VITELLU $>$ vadiel, PEDUCLU > padučo, ME VIDET > ma vido ecc.; PROPINQUI > prakueinti, ven. procazar $>$ prakasá ecc. ${ }^{10}$ Questa seconda tendenza agisce anche nell'intertonica postonica: FEMINA > fimana, FULMINE > foulmano, TURBIDU $>$ túrbado, DIABOLU $>$ giavo ma anche diavalo, ven. nonzolo $>$ nonsalo 'sagrestano' ecc.

I nostri neologismi illustrano entrambe queste tendenze:

/e o > i u/: pineicula, -icula $(<$ pellicola $)$, sipilein $(<$ zeppelin), tilifon $(\mathrm{se}<$ telefón; v. anche av.), tilivision $(<$ televisione $) ;$ bulitur $(<$ bollitore $)$, muturein $(<\operatorname{motorin}(0))$, utumobile, -nobile $(<$ automobile, ma v. anche un po' av.);

/e o $>$ a/: $\quad$ farata $(<$ ferrata $)$, taliefano, -fono $(<$ telefono; la prima forma illustra $/ \mathrm{e}<\mathrm{a} /$ protonico e postonico).

5.5 Altri fenomeni fonetici minori nei neologismi:

- il betacismo: bapur (< vapore) (cfr. VOCE > buz, VULPE > bulpo ecc.);

- l'anaptissi: futubal $(<$ football $)$;

- assimilazione vocalica: nutunobile, utuno-, utumo- (/au $-\mathrm{u}>\mathrm{u}-\mathrm{u} /)$;

- dissimilazione vocalica: natunobile (/au $-\mathrm{u}>\mathrm{a}-\mathrm{u} /) ;^{11}$

- dissimilazione consonantica: -nobile $<$-mobile $(/ \mathrm{m}-\mathrm{b}>\mathrm{n}-\mathrm{b} /)$ pineicula, -icula $<$ pellicola $(1-1>\mathrm{n}-1)$;

- concrezione della $/ \mathrm{n} /$ (probabilmente la $/ \mathrm{n} /$ dell'articolo indefinito): natunobile, nutunobile.

5.6 Non essendoci nel sistema istroromanzo i fonemi / $\breve{\mathrm{z}} \mathrm{ts} \mathrm{dz} /$, essi vengono sostituiti da /s/ risp. /z/: lasá 'lasciare', Rouzisa soprannome (dign.) < croato

10 Mentre /e o > i u/ vale anche per altri dialetti istroromanzi (soprattutto per il dignanense), /e o > a/ sembra limitato quasi esclusivamente al rovignese, assai raro e sporadico altrove. Il comune, in entrambe le tendenze, è la riduzione tendenziale del vocalismo atono alle tre vocali estreme, $/ \mathrm{i}$ a $\mathrm{u} /$. Per l'evoluzione del vocalismo tonico e atono del rovignese rimandiamo a Deanović 1954, per tutti i dialetti istroromanzi a Ive 1900, per il dignanese (ma anche altri dialetti istroromanzi odierni) a Tekavčić 1970 e $1972-1973$.

11 Sia in natunobile che in nutunobile (utumobile, utunobile) la riduzione del dittongo (ad /a/ risp. /u/) postulerebbe una /u/ seguente, dunque la chiusura previa di auto-in autu-, ma riteniamo che i medesimi due fenomeni (assimilazione, dissimilazione) sarebbero immaginabili anche con la /o/ seguente (in tal caso, ovviamente, /o $>\mathrm{u} /$ sarebbe posteriore alla riduzione di /aw/). 
Ružica; seio 'zio', miezo 'mezzo'. Nei nostri neologismi: saparnein, masinigaver, sipilein.

5.7 Infine, vogliamo raggruppare le parole che compaiono in due o più varianti e ricapitolare $\mathrm{i}$ fenomeni fonetici che in esse si possono illustrare:

1. masinigaver, masinegaver: la prima forma esemplifica /e $>\mathrm{i} /$ in protonia;

2. natunobile: chiusura $/ \mathrm{o}>\mathrm{u} /$ in protonia, concrezione della $/ \mathrm{n} /$, dissimilazione del dittongo $/$ aw $/$, dissimilazione della $/ \mathrm{m} /$ in $/ \mathrm{n} /$

nutunobile: chiusura $/ \mathrm{o}>\mathrm{u} /$ in protonia, concrezione della $/ \mathrm{n} /$, assimilazione del dittongo $/$ aw $/$, dissimilazione della $/ \mathrm{m} /$ in $/ \mathrm{n} /$

utumobile: chiusura /o $>\mathrm{u}$ / in protonia, assimilazione del dittongo /aw/

utunobile: come il precedente + dissimilazione della $/ \mathrm{m} /$ in $/ \mathrm{n} /$;

3. pineicula: chiusura $/ \mathrm{e}>\mathrm{i} /$ in protonia e $/ \mathrm{e}>\mathrm{u} /$ in postonia, dittongo /ey/ipercorretto, dissimilazione della prima $/ 1 /$ in $/ \mathrm{n} /$,

pinicula: come il precedente, meno il dittongo /ey/;

4. taliefano: dittongo ipercorretto /ye/, apertura /e $>$ a/ sia in protonia che in postonia

taliefono: dittongo ipercorretto /ye/, apertura $/ \mathrm{e}>\mathrm{a} /$ solo in protonia

tilifon: chiusura /e > i/ in entrambe le sillabe protoniche, se l'accento è su /o/; chiusura nella sola sillaba iniziale, /i/ ipercorretta nella tonica, se l'accento è su di essa.

5.8 A differenza di tutti i fenomeni fin qui analizzati, la caratteristica sostituzione $/ \mathrm{e} \rightarrow \mathrm{o} /$ in posizione finale (CARNE $>$ karno, GRANDE $\mathrm{m}$. sg. $>$ grando, VENDIT $>$ vendo, -MENTE $>$ ment( $r$ )o, SEMPER $>$ SEMPRE $>$ sempro ecc.), ${ }^{12}$ documentata praticamente in tutto il dominio istroromanzo, non appare nel nostro corpus: $i$ termini per 'automobile' conservano tutti la $-e$ lo stesso vale anche per cine (che non presenta, ad. es., neppure il dittongo ipercorretto /ey/).

6. In grandi linee, gli adattamenti formali e il contenuto semantico dei nostri neologismi confermano quanto stabilito già prima per altre componenti alloglotte o comunque non genuine nel rovignese: i neologismi non sono corpi estranei nell'idlioma vivo, ma vengono assimilati e sottoposti alle tendenze del sistema ancora vive ed operanti. Essi sono perciò una parte costitutiva dell'istroromanzo vivo, e l'estensione dello studio ad altri domini ed altri periodi - altrettanti argomenti per ricerche future - fornirà senza dubbio un quadro sostanzialmente identico.

12 Da questa sostituzione sono immuni i termini di riguardo per i genitori (sarpare 'signor padre', namare o dunamare 'signora madre'), i numerali penetrati evidentemente per via della scuola (seinkue, siete / sete, nuove / nove, ğize), e certe voci di carattere più o meno letterario, come grave, (im)puseibile ecc. V. per tutto il problema Tekavčić 1974. 
7. Elenco delle parole analizzate:

auto (v. natunobile, nutunobile, utumobile, utunobile)

bapur

binario

bulitur

cine

curiera

farata

futubal

lavatreice

lietrico

masinegaver (v. masinigaver)

masinigaver (v. masinegaver)

machinario

machineista

mascara

muturein

natunobile (v. auto, nutunobile, utumobile, utunobile)

nutunobile (v. auto, natunobile, utumobile, utunobile)

pineicula (v. pinicula)

pinicula (v. pineicula)

saparnein

sipilein

taliefano (v. taliefono, tilifon)

taliefono (v. taliefano, tilifon)

tilifon (v. taliefano, taliefono)

tilifunada

tilivision

utumobile (v. auto, natunobile, nutunobile, utunobile)

utunobile (v. auto, natunobile, nutunobile, utumobile)

\section{LETTERATURA:}

Alinei 1974: M. Alinei, La struttura del lessico, Bologna.

Deanović 1954: M. Deanović, Avviamento allo studio del dialetto di Rovigno d'Istria, Zagreb.

Diekmann 1983: E. Diekmann, Zur sprachlichen Situation des Rätoromanischen in Graübunden, «Ladinia» VII, pp. 193-209.

Diekmann 1984: E. Diekmann, Zu lexikalischen Problemen des Bündnerromanischen, in: Das Romanische in den Ostalpen (ed. D. Messner), Wien, pp. 309 328. 
Istria Nobilissima: Antologia delle opere premiate, Trieste: Primo concorso d'Arte e di Cultura «Istria Nobilissima», vol. I (1968) - Sedicesimo Concorso d'Arte e di Cultura «Istria Nobilissima», vol. XVI (1983).

Ive 1900: A. Ive, I dialetti ladino-veneti dell'Istria, Strasbourg.

Tekavčić 1970: P. Tekavčić, Sulla molteplicità dei riflessi delle vocali latine nei dialetti istroromanzi, «Revue Roumaine de Linguistique» 15, pp. 223-240.

Tekavčić 1972-1973: P. Tekavčić, Il comune e lo specifico nel dominio istroromanzo, «Studia Romanica et Anglica Zagrabiensia» 33-36, pp. 639-678.

Tekavčić 1974: $\mathrm{P}$. Tekavčić, Interferenze linguistiche istroromanzo-venete: sulle vocali finali nell'istroromanzo, in: Atti del XIV Congresso Internazionale di Linguistica e Filologia Romanza (Napoli 1974), pp. 447-467.

Tekavčić 1983: P. Tekavčić, Osservazioni sulla lingua dei testi istroromanzi contemporanei, in: Linguistica e Dialettologia Veneta, Studi offerti a Manlio Cortelazzo dai colleghi stranieri, Tübingen, pp. 101-111.

Tekavčić 1983b: P. Tekavčić, Problemi di grafia e di trascrizione nei testi istroromanzi, in: Radovi Pedagoškog fakulteta u Rijeci, OOUR nastavne djelatnosti Pula, num. 4, pp. 135-149.

Tekavčić 1984a: P. Tekavčić, Latinizni u pisanim istroromanskim izvorima, «Filologija» 12, pp. 105-121.

Tekavčić 1984b: P. Tekavčić, Le funzioni pragmalinguistiche dei croatismi nei testi rovignesi contemporanei, «Linguistica» 24 , pp. 335-353.

Tekavčić L'elemento tedesco: P. Tekavčić, L'elemento tedesco nel rovignese contemporaneo dell'antologia Istria Nobilissima, in corso di stampa in «Vox Romanica».

Tekavčić Riflessi: Tekavčić, Riflessi di lingue, popoli, culture nella lingua della prosa rovignese contemporanea, uscirà in «Bollettino dell'Atlante Linguistico Mediterraneo» (numero in memoriam di Mirko Deanović).

Sažetak

\section{TEHNIČKI I SRODNI NEOLOGIZMI U SUVREMENOJ ROVINJSKOJ PROZI}

Ovaj se rad nadovezuje na prethodne leksikološke studije pojedinih inojezičnih komponenata u suvremenim književnim tekstovima na rovinjskom dijalektu, objavljenim u antologiji Istria Nobilissima. Dio jezika čine i neologizmi, koje suvremena lingvistika proučava jednako kao i tzv. "čiste«, tj. arhaične, konzervativne sektore jezičnog blaga; dapače, baš neologizmi dokazuju vitalnost jezika. Ovdje se proučava dvadesetak neologizama u zadnjih stotinu godina, s područja prometnih sredstava, različitih sprava, a dijelom i sporta i rata. Nakon analize primjerâ (v. popis u § 7) studira se semantički sadržaj tih riječi (primejenjujući, uz neke preinake, postupak proveden u knjizi M. Alinei, La struttura del lessico), a zatim fonetske adaptacije neologizama, prema fonetskim procesima i pojavama u rovinjskoj (i uopče istroromanskoj) historijskoj fonetici. I te adaptacije dokazuju sa svoje strane da neologizmi nisu strana tijela u današnjem jeziku nego njegov sastavni dio, koji živi zajedno s njim pa ima i određene vrijednosti i funkcije u književnim tekstovima. 